\section{Rituximab and autologous stem-cell transplantation for high-risk diffuse large B-cell lymphoma}

\begin{abstract}
Authors' reply
The results from our randomised phase 3 trial $^{1}$ showed that young patients, affected by high-risk diffuse large B-cell lymphoma (age-adjusted International Prognostic Index score of 2-3), who received abbreviated rituximab-dose-dense chemotherapy plus high dose therapy and autologous stem-cell transplantation compared with patients who received full course rituximab-dose-dense chemotherapy had an improvement in failure-free survival, but not in overall survival.
\end{abstract}

Tetsuya Tanimoto and colleagues commented that the presence of more than one extranodal site of disease might affect the prognosis in young patients with untreated high-risk diffuse large B-cell lymphoma and treated in the rituximab-era. ${ }^{2}$

In our study, ${ }_{1}^{1}$ involving 399 patients younger than 60 years, 127 (32\%) patients had a number of extranodal sites that were more than one at diagnosis, equally distributed into the four randomisation arms (32\% in each group). At the end of the treatment, 208 (76\%) of 272 patients with no or one extranodal site of disease achieved a complete response compared with 87 (69\%) of 127 with more than one extranodal localisation.

In our analysis, patients with more than one extranodal site of disease showed reduced failure-free survival and overall survival compared with those with no or one extranodal site during the first year of observation, but differences disappeared progressively over time (appendix). The failure-free survival at 2 years was $66 \%(95 \% \mathrm{Cl} 60-72)$ for those with more than one extranodal site compared with 66\% (57-74) for those with no or one extranodal site (log-rank test $\mathrm{p}=0.87$ ). The overall survival at 5 years was $76 \%$ (95\% Cl 67-82) for those with more than one extranodal site compared with $78 \%$ (72-83) for those with no or one extranodal site (log-rank test $\mathrm{p}=0.42$ ).

In conclusion, in our randomised phase 3 trial, the number of extranodal sites of disease did not influence the outcome of young patients with highrisk diffuse large B-cell lymphoma with poor prognosis.

$\mathrm{AC}$ is on the advisory board of Celgene; and has received lecture fees from Amgen, Celgene, Janssen, Nanostring, Pfizer, Roche, and Teva. MM is on the advisory board of Roche, Janssen, Celgene, Mundipharma, and Teva; and has received lecture fees from Roche, Celgene, and Janssen. UV is on the advisory board of Roche, Janssen, and Celgene; and has received lecture fees from Roche, Celgene, Janssen, Gilead, and Takeda. AE declares no competing interests.

*Annalisa Chiappella,

Maurizio Martelli, Andrea Evangelista, Umberto Vitolo

achiappella@cittadellasalute.to.it

Department of Haematology, Azienda Ospedaliero Universitaria Città della Salute e della Scienza di Torino, 10126 Turin, Italy (AC, UV); Department of Cellular Biotechnologies and Haematology, Sapienza University, Rome, Italy (MM); and Unit of Clinical Epidemiology, CPO, Azienda Ospedaliero Universitaria Città della Salute e della Scienza di Torino, Turin, Italy (AE)

1 Chiappella A, Martelli M, Angelucci E, et al Rituximab-dose-dense chemotherapy with or without high-dose chemotherapy plus autologous stem-cell transplantation in high-risk diffuse large B-cell lymphoma (DLCL04): final results of a multicentre, openlabel, randomised, controlled, phase 3 study. Lancet Oncol 2017; 18: 1076-88.

2 Glesson M, Cunningham D, Linch D. The role of myeloablation for lymphoma. $N$ EnglJ Med 2014; 370: 575. 